\title{
Low-Complexity Charging/Discharging Scheduling for Electric Vehicles at Home and Common Lots for Smart Households Prosumers
}

\author{
Abbas Mehrabi, Member, IEEE, and Kiseon Kim, Senior Member, IEEE
}

\begin{abstract}
Plug-in electric vehicles are becoming one of indispensable prosumer electronics components for smart households and therefore, their cost efficient energy scheduling is one of the main challenging issues. In the current schemas, the charging and discharging interval of the vehicles are normally announced by the owners in advance leading to the suboptimal profit gain in some situations and hence consumers dissatisfaction. In this paper, we propose an efficient charging/discharging scheduling mechanism for electric vehicles in multiple homes common parking lot for smart households prosumers. The proposed mechanism takes into account the optimal interval allocation considering the instantaneous electricity load and the vehicles request pattern. Based on the data from the vehicles, a mixed optimization model is formulated by the central scheduler which aims to maximize the profit of consumers and is then solved using an effective algorithm. The optimization results are then sent to the system controller determining the interval and energy trading patterns between the power grid and the vehicles. The proposed algorithm has low complexity and ensures the energy satisfaction for all consumers. The performance of the scheduling schema is verified through multiple simulation scenarios.
\end{abstract}

Index Terms-Plug-in Electric Vehicles, Home Stations, Charging and Discharging, Profit Maximization, Consumers Satisfaction.

\section{INTRODUCTION}

Due to the environmental issues such as the increase in $\mathrm{CO} 2$ emissions from high pollution caused by oil dependent vehicles, the penetration of electric vehicles is expected to take a considerable place of indispensable prosumer electronics components [1], [2]. Among various categories of electric vehicles, plug-in electric vehicles have found the most attentions from both research and practical perspectives due to their simpler modeling [3]-[6]. Plug-in electric vehicles which belong to nonperiodic non-real-time appliances [7] have significant role in shaving the electricity load on the grid.

To compensate the instability of power grid caused by unidirectional energy flow, the bidirectional energy flow between the electric vehicles and the grid known as vehicle-to-

\footnotetext{
This research was a part of the project titled Domestic products development of marine survey and ocean exploration equipments, funded by the Ministry of Oceans and Fisheries, and by GIST Research Institute (GRI)

Abbas Mehrabi is with the Department of Computer Science, Aalto University, Espoo, Finland. P.O.Box: 15400, FI-00076 (Email: abbas.mehrabidavoodabadi@aalto.fi ).

Kiseon Kim is with the School of Electrical Engineering and Computer Science, Gwangju Institute of Science and Technology (GIST), P.O.Box. 61005, Gwangju, Korea. (Email: kskim@ gist.ac.kr).
}

grid has been introduced as a viable solution by providing the ancillary services back to the grid [2], [3], [8], [9]. In the context of smart households prosumers electronics, the integration of electric vehicles for charging or discharging will have significant impact on the energy consumption pattern of the appliances except for electric vehicles. Considering the realtime electricity pricing (RTP) model declared by the energy utility company [3], [4], [10], the scheduling of electric vehicles in bidirectional vehicle-to-grid communication has been extensively studied from different perspectives such as minimizing the overall energy cost [3], [8], [10] or maximizing the vehicle consumer satisfaction [5]. He et al. [3] addressed the problem of minimizing the costs for electric vehicles in their charging and discharging scheduling taking into account the RTP pricing model and the internal battery associated costs.

The cost minimization in smart charging scheduling of conservative and green EVs at a single charging station equipped with solar panels has been investigated [11]. Recently, the utilization of cloud computing facilities into the smart grid communications for efficient charging and discharging of electric vehicles has been also studied [12]. From the auxiliary energy resources point of view, Chaudhari et al. [10] have investigated the problem of minimizing the operational costs of charging stations in scheduling of the vehicles by integrating the energy storage system (ESS) into the charging stations. In the context of consumer electronics, Jo et al. [13] introduced the energy cost minimization in scheduling of electronic devices with the integration of auxiliary energy resources and the charging of electric vehicles into the home energy management system (HEMS). The customer convenience is provided through solving a mixed optimization problem with the objective of minimizing the overall costs. The interaction of HEMS with the consumers toward minimizing the electricity cost of home appliances considering both time of use (ToU) and inclining block rate (ICB) pricing models has been also addressed [14]. Wi et al. [15] have studied the problem of scheduling of electric vehicles based on the predicted photovoltaic output and according to the consumer preference during different time periods of the scheduling.

In spite of such extensive study on vehicle-to-grid scheduling, the current existing algorithms assume that the desired interval for charging and discharging of the electric vehicle is provided to the scheduler by the consumer in advance. Furthermore, no attention has been paid to the combination of inclining block rates (ICB) [16] and real-time pricing model (RTP) [17] as a more realistic pricing model which is mostly applicable for 


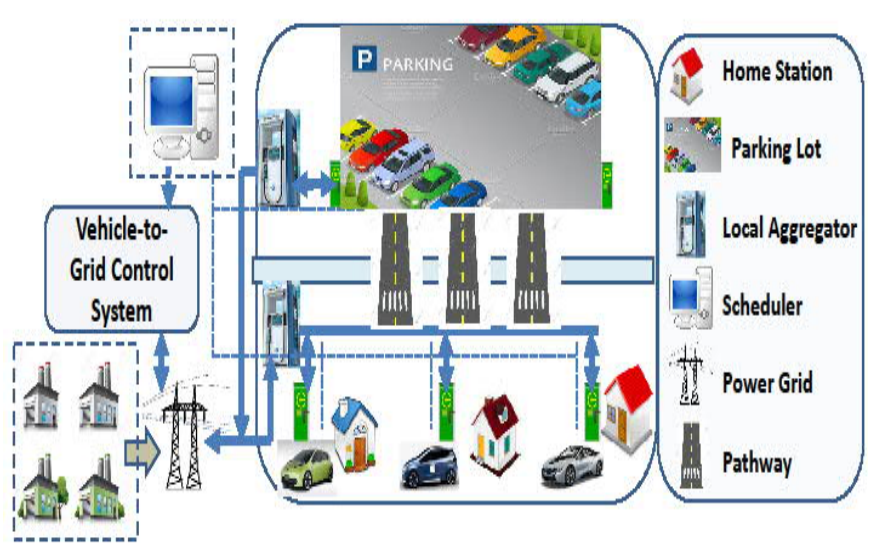

Fig. 1. The framework of vehicle-to-grid system in multiple home stations common parking lot scenario (Dashed and bold lines represent the communication and energy lines, respectively).

charging and discharging of the vehicles for smart households prosumers. Therefore, our main contributions in this work are summarized as follows:

1) Aiming to improve the profit gain for household prosumers, we design an efficient charging/discharging scheduling mechanism for the consumers of electric vehicles in multiple home stations common parking lot scenarios.

2) The proposed system and the scheduling mechanism provide the flexibility of sending the data from the electric vehicles to the central scheduler where a novel mixed optimization model is constructed for the optimal interval allocation and the energy power determination.

3) An efficient algorithm based on the greedy heuristic is then run by the central scheduler to solve the optimization problem. The algorithm has low complexity and ensures the satisfaction of energy requirements for all consumers. The results of the conducted simulations verify the performance of the proposed scheduling mechanism in terms of improving the profit gain for the consumers, better ancillary services for the power grid and reducing the percentage of unserviced vehicles.

The remaining parts of the paper are organized as follows: The proposed system model and its components are described in Section II. The profit maximization methodology and the scheduling algorithm are detailed in Section III. The results of conducted simulations are presented in Section IV and finally Section V concludes the paper.

\section{EleCtRIC Vehicle-TO-GRID TARGET SyStEM}

Fig. 1 illustrates the framework of target vehicle-to-grid system model and its prosumer electronic components. The system facilitates the bidirectional energy flow between the electric vehicles and the power grid through the charging and discharging at home stations and common parking lot. Vehicleto-grid operations are performed under the coordination of local aggregators which are in direct connection with the power grid. The local aggregator coordinates the energy trading between vehicle-to-grid control system and multiple charging or discharging outlets. Upon the request of each vehicle's owner, its data including the arrival/departure times, initial state of charge (SoC) and final energy target are sent through the communication line to the central scheduler. The scheduler then runs an efficient algorithm based on a mixed integer nonlinear programming (MINLP) optimization model to determine the optimal interval for each vehicle as well as the charging or discharging power at each time slot during the allocated interval. The scheduler then sends the scheduling commands to vehicle-to-grid control component of the system to establish the energy flow between the grid and electric vehicles under the coordination of local aggregators. Fig. 2 demonstrates the interaction between the main components of the system toward the determination of optimal charging/discharging interval and energy power allocation for the requesting vehicles.

To analytically model the system and the scheduling algorithm, we use the discrete index $t \in\{1,2, \ldots,|\mathrm{T}|\}$ throughout the paper for the representation of time slot where $|\mathrm{T}|$ is the total number of time slots during the scheduling day. The duration of each time slot is also denoted by $\Delta \mathrm{t}$. Furthermore, the set of consecutive time slots Interval $(a)=\left\{\mathrm{t}_{\mathrm{fa}}, \ldots, \mathrm{t}_{\mathrm{la}}\right\}$ represents the charging or discharging interval of vehicle where $t_{f a}, t_{l a}$ are respectively the starting and ending time slots of the interval. Notation $\mathrm{N}_{\mathrm{t}}$ is also defined which represents the number of plugged-in vehicles to the grid at time slot $t$. Furthermore, in order to avoid the situations where the grid becomes overloaded in the case of charging or underloaded in the case of discharging, the constant $\mathrm{C}_{\max }$ is defined which limits the maximum number of electric vehicles that can be plugged-in to the grid at each time slot. In the following, we describe the main characteristics and the involving entities of the target vehicleto-grid system from the consumers point of view.

\section{A. System Dynamicity}

The system considers the dynamicity in the vehicles arrival and departure such that based on a daily regular pattern, the consumers leave home in the morning at an arbitrary time from a specified interval and depart from their office to reach the home at the evening time. The fleet of electric vehicles for charging and discharging scheduling is denoted by set $\mathrm{M}=\mathrm{M}^{\mathrm{CHG}} \cup \mathrm{M}^{\mathrm{DCG}}$. The vehicles belong to subset $\mathrm{M}^{\mathrm{CHG}}$ are willing to participate in charging operation while those belong to subset $\mathrm{M}^{\mathrm{DCG}}$ participate in only discharging. The arrival and departure times of vehicle $a$ are represented by $\mathrm{A}_{\mathrm{a}}$ and $\mathrm{D}_{\mathrm{a}}$, respectively. Depending on the future trip scheduling of the vehicle, the owner announces its own desired plug-in duration and the departure time to the central scheduler once it decides to participate in vehicle-to-grid program at either home station or common parking lot. Then, based on the instantaneous electricity load on the power grid and by solving a mixed optimization problem, the scheduler determines the optimal charging/discharging interval as well as the amount of energy trading for the requested vehicle. As demonstrated in Fig. 2, the scheduler then sends the scheduling commands to the control system to initiate the vehicle-to-grid operation.

In multiple home stations common parking lot for smart household prosumers, the system considers the continues and regular charging and discharging operations during the allocated interval of the vehicle. For the vehicle with index $a$, lets denote by $\mathrm{B}_{\mathrm{a}}, \mathrm{E}_{\text {ini }}^{\mathrm{a}}$ and $\mathrm{E}_{\text {fin }}^{\mathrm{a}}$ as respectively the battery capacity, the initial stored energy in the battery and the final energy target set by the owner of the vehicle. With maximum 


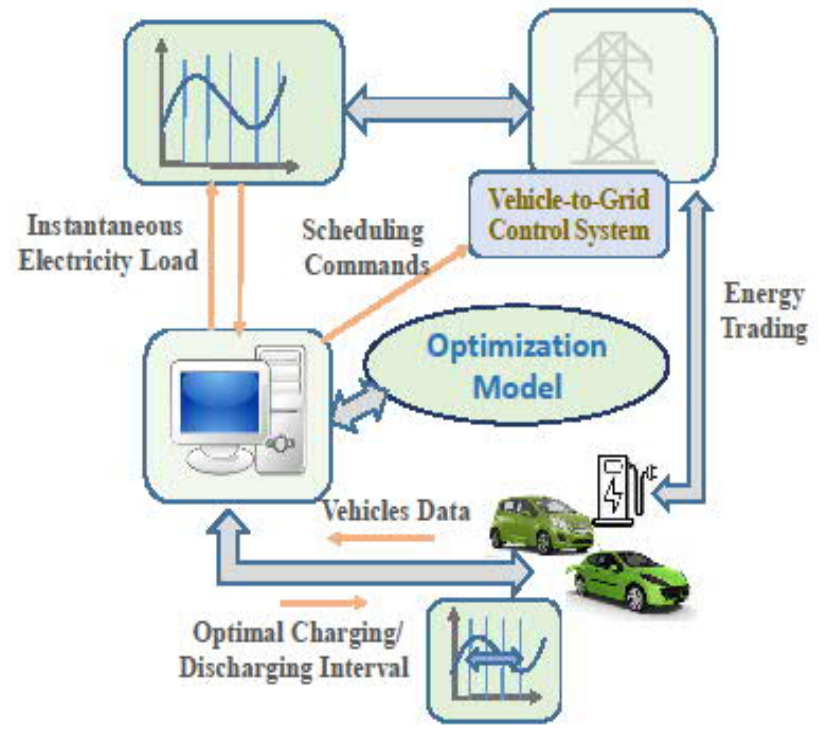

Fig. 2. A schematic view of the interaction between the system components.

feasible charging power $\mathrm{P}_{\max }^{\mathrm{c}}$ and discharging power $\mathrm{P}_{\max }^{\mathrm{d}}$ at each time slot, variable $\mathrm{e}_{\mathrm{at}}$ represents the charging or discharging power allocated to vehicle $a$ at time slot $t$. Also, the constant force of electric motor denoted by $F$ is taken into account for computing the energy consumption during the traveled distance $d$.

\section{B. Consumer Convenience}

The vehicle-to-grid system also provides the consumer convenience in the sense that among the set of electric vehicles in either home stations or common parking lot, those will send their data to the scheduler who are willing to participate in the energy trading depending on the SoC of their battery and the future trip plan.

\section{Household Electricity Pricing}

One of the most important consumer electronics factors in the system model which affects the charging and discharging policy at each plugged-in time slot is the instantaneous household electricity price announced by the energy utility company [4], [10]. Under the theme of smart scheduling and in order to encourage the consumer of electric vehicles to charge and discharge at time slots with low and high electricity demands, respectively, the target system applies the ICB-RTP pricing model with respect to other household appliances. Relation $\mathrm{z}_{\mathrm{t}}=\mathrm{L}_{\mathrm{t}}+\sum_{\forall \mathrm{a} \in \mathrm{M}} \mathrm{e}_{\mathrm{at}}$ states the current load at time slot $t$ where, $\mathrm{L}_{\mathrm{t}}$ is the base electricity loaded on the grid which is from the appliances except for the electric vehicles. Represented by $\mathrm{Z}$, the threshold for electricity load and $\mathrm{P}_{\mathrm{c}}$, the constant price determined by the energy utility company, the ICB-RTP pricing model is described as $\mathrm{P}_{\mathrm{t}}\left(\mathrm{z}_{\mathrm{t}}\right)=\mathrm{P}_{\mathrm{c}}$ for $\mathrm{z}_{\mathrm{t}} \leq \mathrm{Z}$ and $\mathrm{P}_{\mathrm{t}}\left(\mathrm{z}_{\mathrm{t}}\right)=\mathrm{c}_{0}+\mathrm{c}_{1} \mathrm{z}_{\mathrm{t}}$ for $\mathrm{Z}_{\mathrm{t}}>\mathrm{Z}$. Constants $\mathrm{c}_{0}, \mathrm{c}_{1}$ are the coefficients of the linear pricing model [3]. It is also noteworthy to mention that the target system model is easily adoptable to other pricing strategies as well since the optimization model run by the scheduler is independent from the pricing model which is applied.

\section{Consumer Profit}

As the main objective of the scheduling is to maximize the overall profit for the consumers, the obtainable profit for each vehicle should be first analyzed. Regardless of the location, the profit that the consumer of plugged-in vehicle $a$ obtains at time slot $t$ from either charging or discharging is given by:

$$
\operatorname{Profit}(a, \mathrm{t})=\operatorname{Revenue}(a, \mathrm{t})-\operatorname{Cost}(a, \mathrm{t})
$$

As the first term in equation (1), the obtainable revenue at time slot $t$ is computed by integrating over the pricing relation in term of electricity load when the load changes from the current to the modified load after either charging or discharging at time slot $t$. More precisely:

$$
\operatorname{Revenue}(a, \mathrm{t})=\int_{\mathrm{z}_{\mathrm{t}}}^{\mathrm{z}_{\mathrm{t}}+\mathrm{e}_{\mathrm{at}}}\left(\mathrm{c}_{0}+\mathrm{c}_{1} \mathrm{z}_{\mathrm{t}}\right) \mathrm{d}_{\mathrm{z}_{\mathrm{t}}}
$$

On the other side, the incurred cost of the electric vehicle at each time slot is calculated as the battery degradation cost due to high charging or discharging powers, the fluctuation as well as the maintenance costs of the vehicle at that time slot.

$$
\operatorname{Cost}(a, \mathrm{t})=\alpha \mathrm{e}_{\mathrm{at}}^{2}+\beta\left(\mathrm{e}_{\mathrm{at}}-\mathrm{e}_{\mathrm{a}(\mathrm{t}-1)}\right)^{2}+\mathrm{MC}_{\mathrm{a}}
$$

where $\alpha$ and $\beta$ are the coefficients for the battery associated degradation and fluctuation costs [3] and $\mathrm{MC}_{\mathrm{a}}$ is the constant maintenance cost that the owner of vehicle $a$ pays at each time slot. Based on ICB-RTP model, up to the threshold load, the constant price is considered in the computation of revenue. Obviously, the overall obtainable profit at slot $t$ i.e. Profit(t) is the summation of the profit of all plugged-in vehicles at slot $t$.

\section{Profit Maximization Methodology}

As illustrated in Fig. 2, the data from the vehicles are sent to the central scheduler where the mixed optimization problem is first formulated which takes into account the current state of the system. In this section, we present the optimization problem which aims to achieve the scheduling objective and satisfy the set of practical constraints. It should be noted that the optimization problem is presented in the offline form i.e. assuming that the data from all consumers of electric vehicles are available in advance. The section is then followed by the proposed scheduling algorithm.

\section{A. Optimization Model}

Considering the starting time slot and the amount of power at each time slot as respectively the integer and real variables (mixed) to be optimized, the problem of maximizing the overall obtainable profit of vehicles from both charging and discharging is formulated as the following mixed integer nonlinear programming (MINLP) optimization model:

$$
\text { Maximize } \quad \sum_{t=1}^{|T|} \operatorname{Profit}(\mathrm{t})
$$

Subject to:

$$
\begin{gathered}
\mathrm{t}_{\mathrm{fa}}, \mathrm{t}_{\mathrm{la}} \in\{1,2, \ldots,|\mathrm{T}|\}, \quad \mathrm{A}_{\mathrm{a}} \leq \mathrm{t}_{\mathrm{fa}}<\mathrm{t}_{\mathrm{la}} \leq \mathrm{D}_{\mathrm{a}}, \forall a \in \mathrm{M} \\
\mathrm{z}_{\mathrm{t}}=\mathrm{L}_{\mathrm{t}}+\sum_{\forall \mathrm{a} \in \mathrm{M}} \mathrm{e}_{\mathrm{at}}, \forall \mathrm{t} \in\{1,2, \ldots,|\mathrm{T}|\} \\
0 \leq \mathrm{E}_{\mathrm{ini}}^{\mathrm{a}}-\mathrm{F} . \mathrm{d}+\sum_{\mathrm{t} \in \mathrm{S}(\mathrm{t})} \mathrm{e}_{\mathrm{t}} \Delta \mathrm{t} \leq \mathrm{B}_{\mathrm{a}}, \forall a \in \mathrm{M}, \forall \mathrm{t} \in \operatorname{Interval}(a)
\end{gathered}
$$




$$
\begin{aligned}
& \mathrm{E}_{\mathrm{ini}}^{\mathrm{a}}-\mathrm{F} . \mathrm{d}+\sum_{\forall \mathrm{t} \in \text { Interval(a) }} \mathrm{e}_{\mathrm{at}} \Delta \mathrm{t}=\mathrm{r}_{\mathrm{a}} \mathrm{B}_{\mathrm{a}}, \quad \forall a \in \mathrm{M} \\
& \sum_{\forall \mathrm{a} \in \mathrm{M}} \mathrm{I}\left(\mathrm{t}_{\mathrm{fa}} \leq \mathrm{t} \leq \mathrm{t}_{\mathrm{la}}\right) \leq \mathrm{C}_{\max }, \quad \forall \mathrm{t} \in\{1,2, \ldots,|\mathrm{T}|\} \\
& \mathrm{t}_{\mathrm{la}}=\mathrm{t}_{\mathrm{fa}}+|\operatorname{Interval}(a)|, \quad \forall a \in \mathrm{M}, \\
& 0 \leq \mathrm{e}_{\mathrm{at}} \leq \mathrm{P}_{\mathrm{max}}^{\mathrm{c}}, \quad \forall a \in \mathrm{M}^{\mathrm{CHG}}, \forall \mathrm{t} \in \operatorname{Interval}(a) \\
& 0 \leq \mathrm{e}_{\mathrm{at}} \leq \mathrm{P}_{\max }^{\mathrm{d}}, \quad \forall a \in \mathrm{M}^{\mathrm{DCG}}, \forall \mathrm{t} \in \operatorname{Interval}(a)
\end{aligned}
$$

In the above model, variables $t_{\mathrm{fa}}, \mathrm{e}_{\mathrm{at}}$ are the integer and real decision variables, respectively. Constraint (5) ensures that the starting time slot of charging or discharging interval of the vehicle does not exceed the ending time slot. Constraint (7) enforces that the stored energy in the battery of the vehicle at the end of each time slot must be non-negative and less than its battery capacity where $\mathrm{S}(\mathrm{t})$ is the discrete set of consecutive slots before time slot $t$. Equality (8) ensures that for each vehicle, the final energy including the initial energy, consumed energy during the traveled distance between home/office and the summation of charging or discharging energies must satisfy the desired target set by the owner of the vehicle which is $r_{a}$ fraction of battery capacity. Constraint (9) imposes the constraint on the maximum number of plugged-in vehicles per time slot at each location and (10) determines the ending time slot in term of the starting time slot and the size of charging or discharging interval. Here, function $I\left(t_{\mathrm{fa}} \leq t \leq t_{\mathrm{la}}\right)$ is $0-1$ indicator function such that for time slot $t, \mathrm{I}\left(\mathrm{t}_{\mathrm{fa}} \leq \mathrm{t} \leq \mathrm{t}_{\mathrm{la}}\right)=1$ if $\mathrm{t}_{\mathrm{fa}} \leq \mathrm{t} \leq \mathrm{t}_{\mathrm{la}}$ and $\mathrm{I}\left(\mathrm{t}_{\mathrm{fa}} \leq \mathrm{t} \leq \mathrm{t}_{\mathrm{la}}\right)=0$, otherwise. Finally, constraints (11) and (12) specify the maximum allowed charging and discharging powers at each time slot.

\section{B. Scheduling Algorithm}

At the next phase, the scheduler needs to run an efficient algorithm in order to determine the starting time slot as well as the amount of charging or discharging power of the vehicle at each time slot of the allocated interval. A simple yet efficient algorithm based on greedy heuristic is developed for the scheduling problem which has low complexity and ensures the satisfaction of energy requirements of all consumers. The body of the algorithm named OptimalInterval Allocation has been shown in Algorithm 1.

For the case of charging, in an optimal scheduling, the charging demand of the consumers must be shifted to the time intervals with low electricity demand on the grid i.e. time slots with cheap price while for discharging, the discharged load must be shifted to time intervals with high price. With initial base load on the grid, for each vehicle in either home stations or parking lot, the proposed algorithm starts from the time slot of arrival and investigates all sets of consecutive time slots with length equal to the size of vehicles interval. The time interval which has available space for the current vehicle as well as it has the least and the most average load among all possible intervals is chosen for respectively charging and discharging operation of the vehicle. As an illustrative example, Fig. 3 shows the allocated charging interval according to the electricity base load. In contrast to the fixed interval allocation in which the charging interval is announced by the consumer in

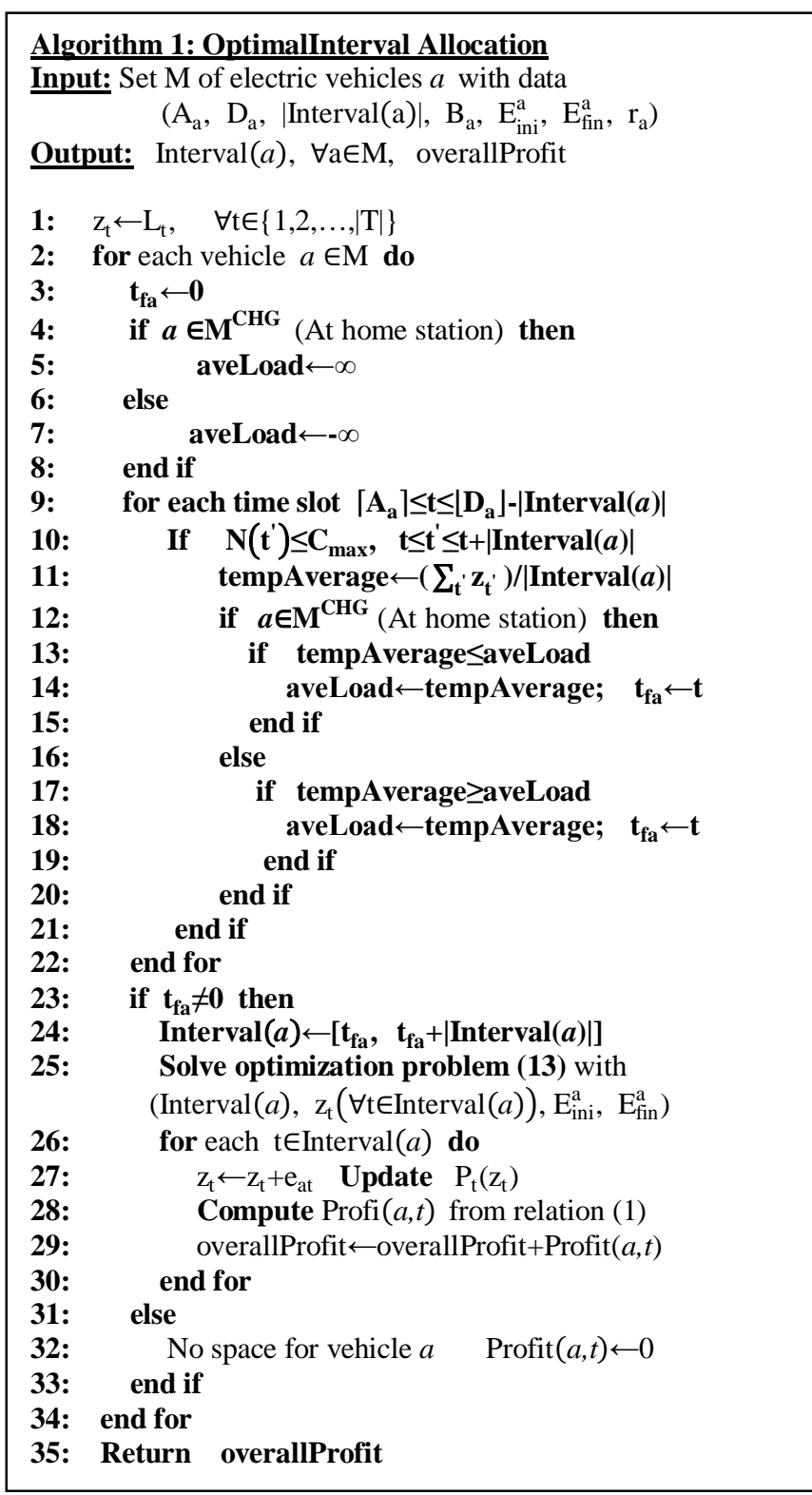

advance, in our system, the scheduler runs the proposed algorithm which assigns the interval with lowest average load according to the instantaneous electricity load on the grid. Updating the price as mentioned in line 27 of the algorithm is also performed according to ICB-RTP model. It should be also mentioned that although the proposed algorithm allocates the locally optimal interval for each electric vehicle, it does not incur high complexity since the interval size of the vehicle is normally small compared to the total number of time slots. After the command was sent to vehicle $a$ determining the interval during which the vehicle should be plugged-in, the scheduler then solves the following optimization to determine the amount of charging or discharging powers at each time slot which will be exchanged between the vehicle and the grid.

$$
\text { Minimize } \sum_{\forall \mathrm{t} \in \operatorname{Interval}(a)}\left(\mathrm{z}_{\mathrm{t}}+\mathrm{e}_{\mathrm{at}}-\overline{\mathrm{z}}\right)^{2}
$$

where $\bar{z}$ is the average of load during the vehicle's interval. Note that the above minimization problem is subject to the 


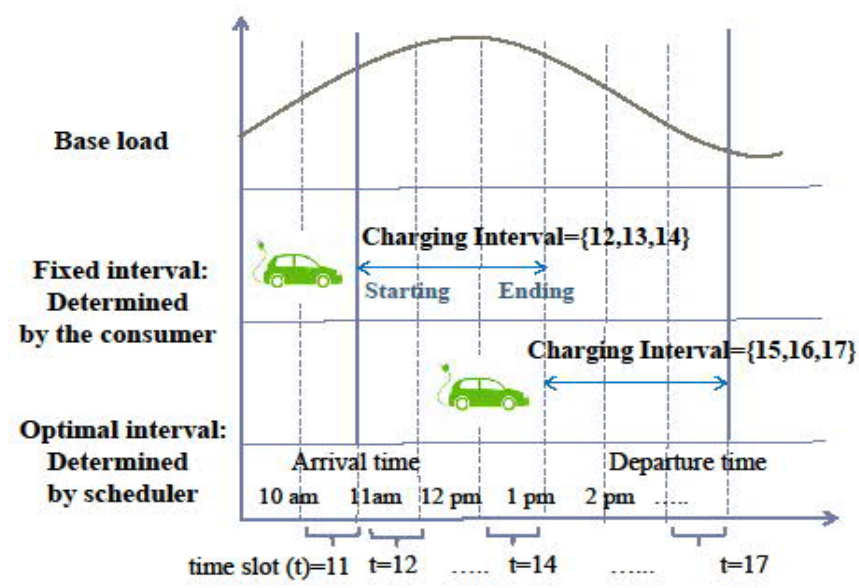

Fig. 3. Comparison between two interval scheduling schema.

constraints (7), (8), (11) and (12). According to (13), the charging or discharging load of the vehicle at each time slot is determined such that the overall deviation of the modified load from the average one is minimized. In fact, the aim of scheduler in solving this optimization problem for power determination is to provide the load flatting on the power grid which is one of the main objectives of the vehicle-to-grid system. Once the charging or discharging powers $e_{a t}$ are determined, the share of profit that vehicle $a$ obtains from its charging or discharging is computed using the relation (1). Meanwhile, the algorithm also updates the overall obtainable profit from all the vehicles plugged-in to the system up to the current time.

\section{Consumer Satisfaction and Complexity}

As mentioned earlier in the system model, the satisfaction of energy requirements of all the vehicles and the low computational complexity are two noticeable features of the proposed scheduling mechanism. Consumer satisfaction is guaranteed by applying the constraint (8) in the optimization problem (13) which is run by the scheduler. In other words, this constraint ensures that the summation of charging or discharging powers during the allocated time interval of the vehicle meets its final energy target. Since this optimization is invoked for each vehicle' request, therefore, the proposed algorithm guarantees the satisfaction of energy requirements for all the vehicles in the system regardless of their location.

From the complexity point of view, it is noticed that at either home station or parking lot, finding the best time interval for each electric vehicle in the greedy manner performs with time complexity of $\mathrm{O}\left(|\mathrm{T}|^{2}\right)$ in the worst case. Furthermore, since for each time slot, the computation of profit according to relation (1) takes $\mathrm{O}(1)$ time, therefore, the last for loop is performed with time complexity of order $\mathrm{O}(|\mathrm{T}|)$. Considering $\mathrm{t}_{\mathrm{opt}}$ as the required computation time for solving the optimization problem (13), with $m$ available electric vehicles in the system, running the algorithm OptimalInterval Allocation results in the worst case time complexity of order $\mathrm{O}\left(\mathrm{m}\left(|\mathrm{T}|^{2}+\mathrm{t}_{\text {opt }}\right)\right)$. Since the allocated interval for each vehicle is normally short, therefore, the overall low complexity of the algorithm makes it practically feasible for smart household prosumers.

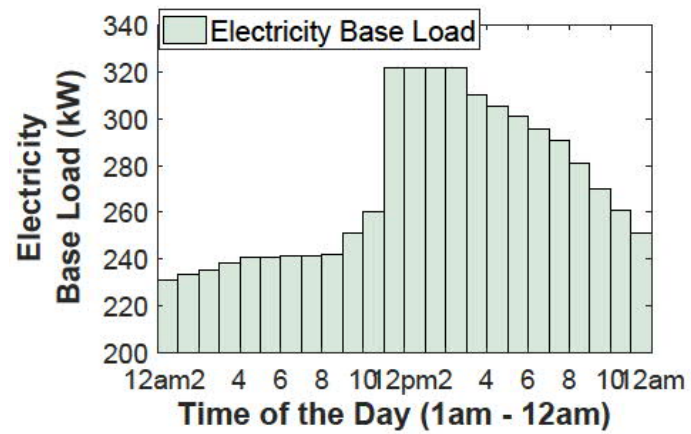

Fig. 4. An adopted typical base load during the summer day [3].

\section{Performance Evaluation}

In this section, we implement the proposed scheduling mechanism for an instance of multiple home stations common parking lot scenario. The fixed interval allocation strategy [11] was adopted to compare the performance of our scheduling algorithm against. Using FixedInterval Allocation algorithm, the starting time slot for vehicle-to-grid operation of the vehicles are deterministic and are announced by the consumers in advance.

\section{A. System Modeling and Simulation Setting}

We first describe the simulation scenario and the settings of the system parameters for the scheduling of a fleet of electric vehicles in multiple home stations common parking lot for smart household prosumers. The charging/discharging scheduling is performed during one day time duration with $\Delta t=1 \mathrm{~s}$ as the duration of each time slot. For the vehicles commuting pattern, the arrival times of the vehicles to the office parking lot and home stations are uniform random values with the density of respectively Unif[7am,10am] and Unif[6pm, 9pm]. A randomly chosen value from the uniform interval Unif[6hours, 9hours] is also considered for the plug-in duration of each vehicle at either home station or common parking lot. During the night time when there is normally low electricity load from home appliances, the system assumes the participation of $80 \%$ of the consumers in charging their electric vehicles while the remaining $20 \%$ involve in discharging operation. In contrast, during the day times, the participation of $80 \%$ of consumers is for discharging while the other $20 \%$ charge their vehicles. As default, the maximum plug-in capacity of $\mathrm{C}_{\max }=100$ is considered for each time slot at each location. As the alternative scheduling strategy, the fixed interval allocation strategy assigns the starting and ending time slots of the vehicles' interval with some random values chosen between the arrival and departure times of the vehicle.

On the vehicle side, an ideal battery capacity of $B_{a}=100 \mathrm{~kW} . \mathrm{h}$ and the maximum charging and discharging powers of respectively $\mathrm{P}_{\max }^{\mathrm{c}}=15 \mathrm{~kW}$ and $\mathrm{P}_{\max }^{\mathrm{d}}=10 \mathrm{~kW}$ at each time slot are considered for the electric vehicles. This charging power corresponds to the charging rate of $15 / 100=0.15 \mathrm{C}$ [18]. As default, the identical electric motor force of $\mathrm{F}=5 \mathrm{~kW} . \mathrm{h} / \mathrm{km}$ is considered for all the vehicles and the traveling distance between the home of consumer and the office is randomly chosen from the uniform interval Unif[2km,3km]. The final energy target is set by the consumer while the initial energy 

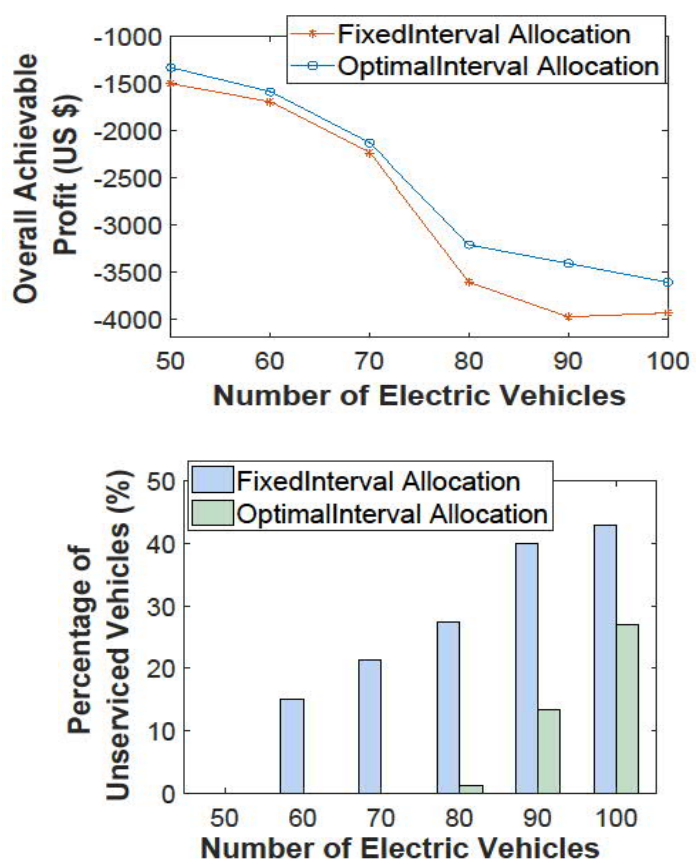

Fig. 5. Comparison between interval scheduling algorithms in term of overall achievable profit (top) and the percentage of unserviced vehicles (bottom).

stored in the battery of the vehicle depends on the final energy at the previous location minus the energy consumed during the travelled distance to the current location. As an instance of the electricity price determined by the energy utility company, for the verification of the scheduling algorithm, the constant electricity load threshold $\mathrm{Z}=250 \mathrm{~kW}$ and constant price $\mathrm{P}_{\mathrm{c}}=1 \$ / \mathrm{kW} . \mathrm{h}$ are used in ICB pricing model and the coefficients $\mathrm{c}_{0}=0.002 \$$ and $\mathrm{c}_{1}=0.004 \$ / \mathrm{kW}$ for RTP model. The battery degradation and fluctuation parameters $\alpha=0.001$ and $\beta=0.002$ [3] are also considered. As shown in Fig. 4, an adopted typical base load of other household appliances during one summer day [3] is also used in the system.

\section{B. System Scalability}

In the first part of simulations, we study the performance of the proposed charging/discharging mechanism considering the scalability of the system when the number of electric vehicles changes from 50 to 100 . Its performance is evaluated in terms of the obtainable profit for the consumers, the percentage of the vehicles which lose their service (unserviced) and the smartness of the schema in energy interval allocation.

The result of comparing two interval allocation algorithms in term of the overall obtainable profit for the consumers of electric vehicles during one day vehicle-to-grid operation has been shown in Fig. 5 (top). As we can see from the result, for different number of vehicles, the OptimalInterval Allocation outperforms FixedInterval Allocation in term of the overall profit that consumers obtain from both charging and discharging. For this simulation instance, the average improvement of about $10 \%$ in profit is achieved using the proposed algorithm. Furthermore, it is also observed that the improvement gap increases as more number of vehicles are plugged-in to the system. The reason is that by increasing the

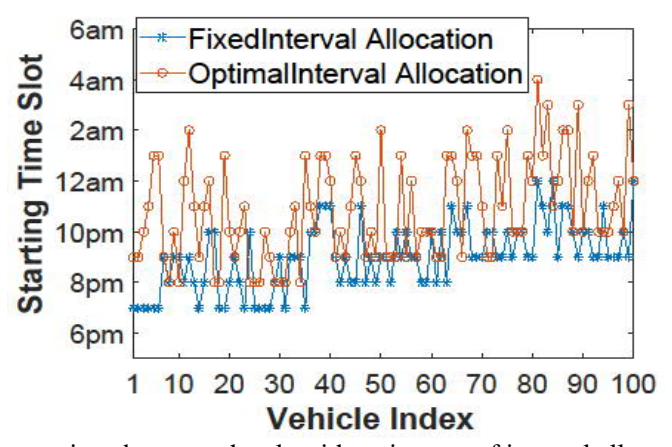

Fig. 6. Comparison between the algorithms in term of interval allocation.

number of vehicles, larger volume of charging and discharging loads are concentrated on time slots with respectively high and low electricity demands when FixedInterval Allocation strategy is applied for the scheduling process.

Next, we have investigated the impact of maximum vehicle capacity at each time slot on the percentage of consumers which do not get the charging service. In fact, with low vehicle capacity at each slot, depending on the interval allocation strategy, some of the consumers may not be able to get their service upon sending the request. With maximum capacity of 40 vehicles at each time slot, the percentage of unserviced vehicles for the charging operation have been shown in Fig. 5 (bottom). As the results confirm for different number of vehicles, OptimalInterval Allocation algorithm results in plugging more number of electric vehicles by looking for the most appropriate time interval with available space. As for this simulation instance, in average, about $17.55 \%$ reduction in the percentage of unserviced vehicles is observed using the proposed algorithm compared to FixedInterval Allocation.

We have been also interested to compare two interval allocation algorithms in term of determining the starting time slot for the charging operation of each consumer. The result of this comparison for charging of 100 electric vehicles during the time interval 6pm until 6am has been shown in Fig. 6. As we can see before $8 \mathrm{pm}$ where the base load is slightly high, the proposed algorithm does not assign the starting time slot of any vehicle while, fixed interval allocation strategy allocates the starting time slot of about $16 \%$ of vehicles before this time in an uncontrolled manner. In contrast, the proposed algorithm allocates the starting time slots of about $42 \%$ of electric vehicles after 12am when the base load is low which confirms the superiority of the proposed algorithm compared to its competitor in term of smart interval allocation.

\section{Ancillary Services}

In the next part, we evaluate the performance of the proposed algorithm in term of ancillary services provided to the power grid. For this simulation, 20 number of vehicles are considered for the charging and discharging operations. The results of comparing algorithms in term of valley filling i.e. load shifting during the time interval $[4 \mathrm{am}, 12 \mathrm{pm}]$ and peak load reduction during the time interval [1pm, 9pm] have been shown in Fig. 7. The root mean square deviation ( $r m s d$ ) with the following definition is used as the metric for measuring the valley filling during the given time interval $t_{1} \leq t \leq t_{2} \quad[6]$ : 

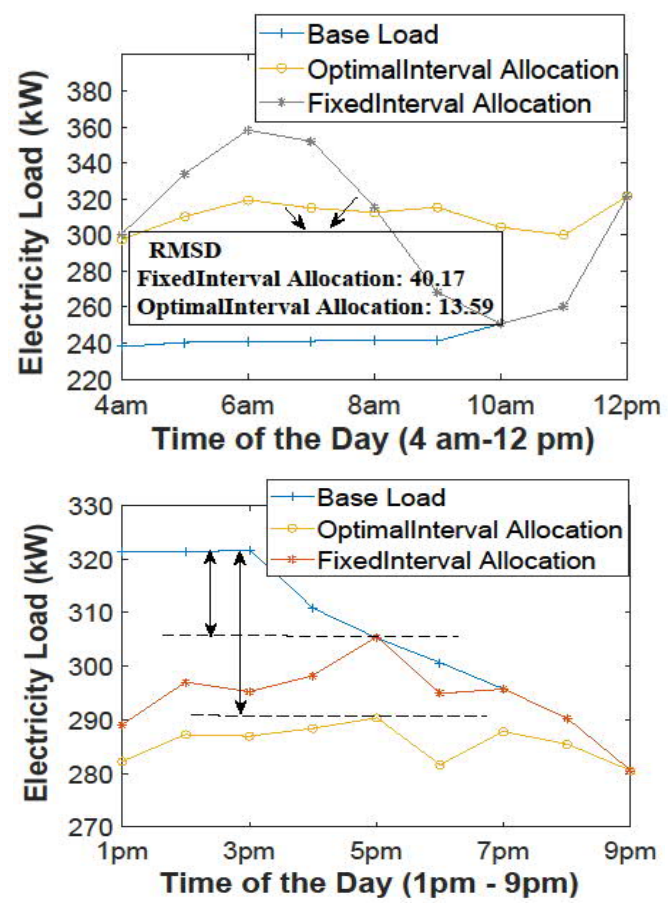

Fig. 7. Comparison between algorithms in term of valley filling (top) and peak load reduction (bottom).

$$
\operatorname{rmsd}=\sqrt{\sum_{\mathrm{t}_{1} \leq t \leq \mathrm{t}_{2}}\left(\mathrm{z}_{\mathrm{t}}-\max \left(\mathrm{L}_{\mathrm{t}}\right)\right)^{2} /\left(\mathrm{t}_{2}-\mathrm{t}_{1}\right)}
$$

As we can see from Fig. 7 (top), the OptimalIntervall and Fixed- Interval allocation algorithms achieve the rmsd values of about 13.59 and 40.17, respectively, confirming that the proposed interval allocation algorithm outperforms significantly FixedInterval allocation in term of load shifting. The reason is that the proposed algorithm allocates time intervals for the consumers in a controlled manner such that their charging load is shifted to the time intervals with low electricity demand on the power grid. With the same number of electric vehicles, we have also compared both interval allocation strategies in term of peak load reduction by adopting the following relation as a metric for peak reduction (PR) during the given time interval $\mathrm{t}_{1} \leq \mathrm{t} \leq \mathrm{t}_{2}[6]$ :

$$
\operatorname{PR}(\%)=\frac{\max \left(\mathrm{L}_{\mathrm{t}}\right)-\max \left(\mathrm{z}_{\mathrm{t}}\right)}{\max \left(\mathrm{L}_{\mathrm{t}}\right)} \times 100
$$

As we can see from the result in Fig. 7 (bottom) for the time period $[1 \mathrm{pm}, 9 \mathrm{pm}]$, the proposed algorithm shows better performance in term of the percentage of peak reduction. For this simulation instance, the optimal and fixed interval allocation strategies reduce the peak load during the aforementioned time period for about $9.7 \%$ and 5\%, respectively. Similar to the case of charging, the proposed algorithm takes into account the current electricity load on the grid and allocates the discharging intervals for the consumers in a controlled way. It is also noteworthy to mention that by penetrating more number of electric vehicles, further peak load reduction can be obtained using the proposed algorithm.

\section{Effect of Electric Motor Force}

We have also investigated the impact of one of the main components of the electric vehicles on its performance in term

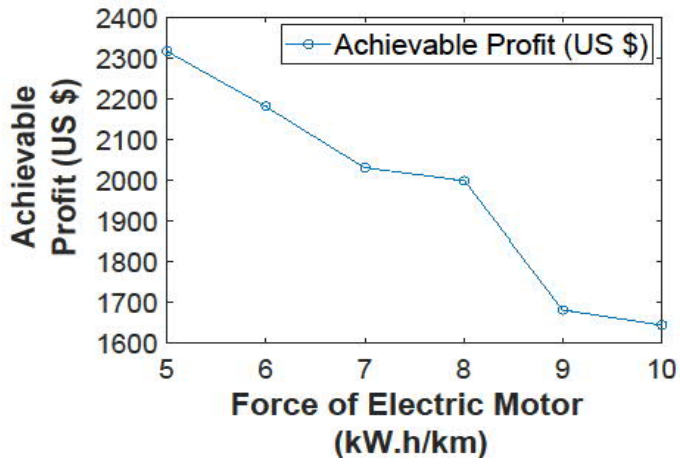

Fig. 8. Impact of motor force on the obtainable profit (\$).

of the achievable profit for the consumers. With 100 electric vehicles, the force of motor is increased from 5 to $10 \mathrm{~kW} . \mathrm{h} / \mathrm{km}$ and corresponding to each motor force, the overall obtainable profit from discharging has been shown in Fig. 8. As we see, the overall profit decreases when the force of electric motor increases. This is because with fixed distance from the home to the parking lot, the vehicles consume more energy when the force of motor increases, therefore, lesser volume of energy will return back to the power grid which in turn yields the reduction in the achievable profit for the consumers.

\section{CONCLUSION}

This paper proposes a low-complexity charging and discharging interval scheduling for electric vehicles in multiple home stations common parking lot scenarios for smart household prosumers. The scheduling objectives are to maximize the profit of all consumers of electric vehicles, provide the ancillary services to the power grid as well as satisfying the energy requirements of all consumers. Based on the instantaneous electricity price and the data from the vehicles, a novel mixed optimization model is first constructed by the central scheduler with the aim of maximizing the overall profit of the consumers. Running an efficient scheduling algorithm at the scheduler, the charging/discharging interval as well as the amount of energy power at each time slot of the interval are determined for each requesting vehicle. The low complexity of the algorithm and the guarantee on satisfying the energy requirement of all consumers makes the algorithm practically attractive for real-word deployment by smart household prosumers.

The results of our simulation instances reveal that the proposed algorithm outperforms the conventional fixed interval allocation mechanism by in average $42 \%$ of electric vehicles in term of smart interval allocation as well as achieving in average about 10\% improvement in overall obtainable profit for the consumers. It is also observed that in term of ancillary services, the proposed algorithm achieves more peak load reduction and yields significant benefits in term of valley filling. The algorithm also efficiently utilizes the available time intervals such that the percentage of unserviced consumers noticeably decreases.

\section{REFERENCES}

[1] A. C. Luna, N. L. Diaz, M. Graells, J. C. Vasquez, and J. M. Guerrero,, "Cooperative energy management for a cluster of households prosumers", IEEE Trans. Consum. Electron., vol. 62, no. 3, pp. 235-242, Aug. 2016. 
[2] S. Pal, and R. Kumar, "Electric vehicle scheduling strategy in residential demand response programs with neighbor connection", IEEE Trans. Ind. Informat., vol. 14, no. 3, pp. 980-988, Mar. 2018.

[3] Y. He, B. Venkatesh, and L. Guan, "Optimal scheduling for charging and discharging of electric vehicles," IEEE Trans. Smart Grid, vol 3, no, 3, pp. 1095-1105, Sep. 2012.

[4] M. Sung, and Y. Ko, "Machine-learning-integrated load scheduling for reduced peak power demand," IEEE Trans. Consum. Electron., vol. 61, no. 2, pp. 167-174, May 2015.

[5] C. K. Wen, J. C. Chen, J. H. Teng, and P. Ting, Decentralized plug-in electric vehicle charging selection algorithm in power systems", IEEE Trans. Smart Grid, vol 3, no 4, pp. 1779-1789, Dec. 2012.

[6] Z. Wang, and S. Wang, "Grid power peak shaving and valley filling using vehicle-to-grid systems," IEEE Trans. Power Del., vol 28, no 3, pp. 18221829, Jul. 2013.

[7] Xin Liu, Liviu Lvanescu, Rui Kang, and Martin Maier, "Real-time household priority scheduling algorithm based on prediction of renewable source availability," IEEE Trans. Consum. Electron., vol. 58, no. 2, pp. 318-326, May. 2012.

[8] E. Sortomme, and M. A. El-Sharkawi, "Optimal scheduling of vehicle-togrid energy and ancillary services", IEEE Trans. Smart Grid, vol. 3, no 1, pp. 351-359, Mar. 2012.

[9] E. Yao, V. W. S. Wong, and R. Schober, "Robust frequency regulation capacity scheduling algorithm for electric vehicles," IEEE Trans. Smart Grid., vol. 8, no. 2, pp. 984-997, Mar. 2017.

[10] K. Chaudhari, A. Ukil, K. N. Kumar, U. Manandhar, and S. K. Kollimalla, "Hybrid optimization for economic deployment of ESS in PV-integrated EV charging stations," IEEE Trans. on Ind. Informat., vol. 14, no. 1, pp. 106-116, Jan. 2018.

[11] W. Tushar, C. Yuen, S. Huang, D. B. Smith, and H. V. Poor, "Cost minimization of charging stations with photovoltaics: an approach with EV classification", IEEE Trans. Intell. Transp. Syst., vol. 17, no. 1, pp. 156-169, Jan. 2016.

[12] D. A. Chekired, and L. Khoukhi, "Smart grid solution for charging and discharging services based on cloud computing scheduling," IEEE Trans. Ind. Informat., vol. 13, no. 6, pp. 3312-3321, Dec. 2017.

[13] H. C. Jo, S. Kim, and S-K. Joo, "Smart heating and air conditioning scheduling method incorporating customer convenience for home energy management system", IEEE Trans. Consum. Electron., vol. 59, no. 2, pp. 316-322, May 2013.

[14] I-Y Joo, and D-H. Choi, "Optimal household appliance scheduling considering consumer's electricity bill target", IEEE Trans. Consum. Electron., vol. 63, no. 1, pp. 19-27, Feb. 2013.

[15] Young-Min Wi, Jong-Uk Lee, and Sung-Kwan Joo, "Electric vehicle charging method for smart homes/buildings with a photovoltaic system," IEEE Trans. Consum. Electron., vol. 59, no. 2, pp. 323-328, May 2013.

[16] J. Han, M. Choi, I. Lee, and S. H. Kim, "Photovoltaic energy sharing system in a multifamily residential house to reduce total energy costs", IEEE Int. Conf. Consum. Electron. (ICCE), pp. 1-2, Jan. 2016.

[17] H. Mohsenian-Rad, and A. Leon-Garcia, "Optimal residential load control with price prediction in real-time electricity pricing environments," IEEE Trans. Smart Grid, vol. 1, no. 2, pp. 120-133, Sep 2010.

[18] P. Fernandez, T. G. S. Roman, R. Cossent, C. M. Domingo, and P. Fras, "Assessment of the impact of plug-in electric vehicles on distribution networks", IEEE Trans. Power Sys., vol. 26, no. 1, pp. 206-213, Feb. 2011.

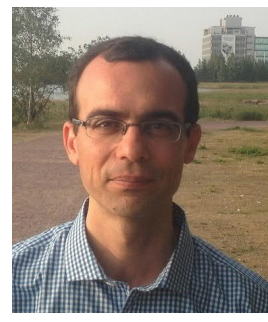

Abbas Mehrabi is Postdoctoral fellow at department of computer science, Aalto University, Espoo, Finland. He received his $\mathrm{Ph} . \mathrm{D}$ in 2017 from the school of electrical engineering and computer science at Gwangju Institute of Science and Technology, Gwangju, South Korea where he was one of the recipients of $\mathrm{Ph} . \mathrm{D}$ graduation award. He also obtained his M.Sc in 2010 and B.Sc in 2008 in computer engineering from respectively Azad University, South Tehran and Shahid Bahonar University of Kerman, Iran.

His main research interests include quality of experience optimization and resource allocation for multimedia services in mobile edge computing environments, energy efficient mobile computing and scheduling/planning problems in smart grids. He is a member of IEEE.

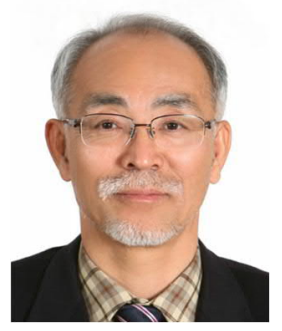

Kiseon Kim received the B.Eng. and M.Eng. degrees in electronics engineering from Seoul National University, Seoul, South Korea, in 1978 and 1980, respectively, and the $\mathrm{Ph} . \mathrm{D}$. degree in electrical engineering systems from the University of Southern California at Los Angeles, Los Angeles, CA, USA, in 1987. From 1988 to 1991, he was with Schlumberger, Houston, TX, USA. From 1991 to 1994, he was with the Superconducting Super Collider Laboratory, Waxahachie, TX, USA. In 1994, he joined the Gwangju Institute of Science and Technology, Gwangju, South Korea, where he is currently a Professor.

His current research interests include wideband digital communications system design, sensor network design, analysis and implementation both at the physical and at the resource management layer, and biomedical application design. He is a member of the National Academy of Engineering of Korea, Fellow IET, Senior Editor of the IEEE SENSORS JOURNAL and Senior member of IEEE. 